\title{
Microstructural Observation with MicroCT and Histological Analysis of Human Alveolar Bone Biopsy from a Planned Implant Site: A Case Report
}

\author{
Emi Yamashita-Mikami ${ }^{1}$, Mikako Tanaka ${ }^{1, *}$, Naoki Sakurai ${ }^{1}$, Kazuho Yamada $^{2}$, Hayato Ohshima ${ }^{3}$, \\ Shuichi Nomura ${ }^{1}$ and Sadakazu Ejiri ${ }^{4}$ \\ ${ }^{I}$ Division of Comprehensive Prosthodontics, Niigata University Graduate School of Medical and Dental Sciences, \\ Niigata, Japan \\ ${ }^{2}$ Oral Implant Clinic, Niigata University Medical and Dental Hospital, Niigata, Japan \\ ${ }^{3}$ Division of Anatomy and Cell Biology of the Hard Tissue, Niigata University Graduate School of Medical and Dental \\ Sciences, Niigata, Japan \\ ${ }^{4}$ Department of Oral Anatomy, Asahi University School of Dentistry, Gifu, Japan
}

\begin{abstract}
The subject was a 53-year-old male. An alveolar bone sample was obtained from the site of the lower left first molar, before dental implant placement. Although the details of the trabecular structure were not visible with conventional computed tomography, micro-computed tomography (microCT) three-dimensional images of the alveolar bone biopsy sample showed several plate-like trabeculae extending from the lingual cortical bone. Histological observations of the bone sample revealed trabeculae, cuboidal osteoblasts, osteoclasts and hematopoietic cells existing in the bone tissue at the implantation site. Bone metabolic markers and calcaneal bone density were all within normal ranges, indicating no acceleration of the patient's bone metabolism.

Using microCT, and histological and histomorphometrical techniques, a great deal of valuable information about the bone tissue was obtained from a biopsy sample extracted from the patient's planned implant site.
\end{abstract}

Keywords: Bone biopsy, bone micro-structure, dental implant, histomorphometry, human alveolar bone, microCT.

\section{INTRODUCTION}

Alveolar bone quality at a dental implant site has been reported to have a great impact on the osseointegrative success or failure of the implant, so several reports have focused on bone quality. The risk of implant failure has been reported to be particularly high when bone quality is poor [1$3]$. In order to facilitate prognosis, alveolar bone quality at implant sites has been divided into four clearlydistinguishable classifications visible on conventional computed tomography (CT) images at preoperative inspection [4]. However, with respect to the image quality obtainable with conventional $\mathrm{CT}$, the problem remains that thin trabeculae are not visible on such CT images. In recent years, the cone-beam computed tomography (CBCT) has been increasingly used in dentistry. The CBCT has the advantages of cost-effectiveness, higher resolution, and less radiation exposure than conventional CT. However, its resolution is still not sufficient to confidently identify fine trabeculae, and beam-hardening artifacts still remain as problems to be

*Address correspondence to this author at the Division of Comprehensive Prosthodontics, Department of Tissue Regeneration and Reconstruction, Niigata University Graduate School of Medical and Dental Sciences, 2-5274 Gakkocho-dori, Chuo-ku, Niigata 951-8514, Japan; Tel: +81-25-227-2892; Fax: +81-25-229-3454; E-mail: mikako@dent.niigata-u.ac.jp solved [5]. Other less-popular methods to quantify and qualify bone quality have included trabecular patterns on preoperative radiographs [6], the cutting resistance technique [7], resonance frequency analysis [8] and the hand-felt perceptions of drilling resistance [9]. These methods are however unable to provide exact data with respect to bone tissue characteristics, and it has been difficult to establish clear classification definitions using these methods [10].

In this case report, an alveolar bone sample was extracted from a patient's implant recipient site and its bone tissue was directly assessed using micro-computed tomography (microCT), and histological and histomophometric techniques, in order to obtain precise, detailed information (including bone cell information) from this sample, with respect to bone remodeling and bone volume.

\section{CASE PRESENTATION}

The patient was a 53-year-old male. About 30 years previously, his lower left first molar had been extracted at a private dental clinic following severe dental caries, with a fixed dental bridge having been constructed in the region of the missing tooth. Approximately fourteen years later in 1991, a new fixed bridge was constructed at the same site, at our hospital's dental clinic. In 2005, the patient returned, and requested implant treatment to replace the bridge (Fig. 1). 


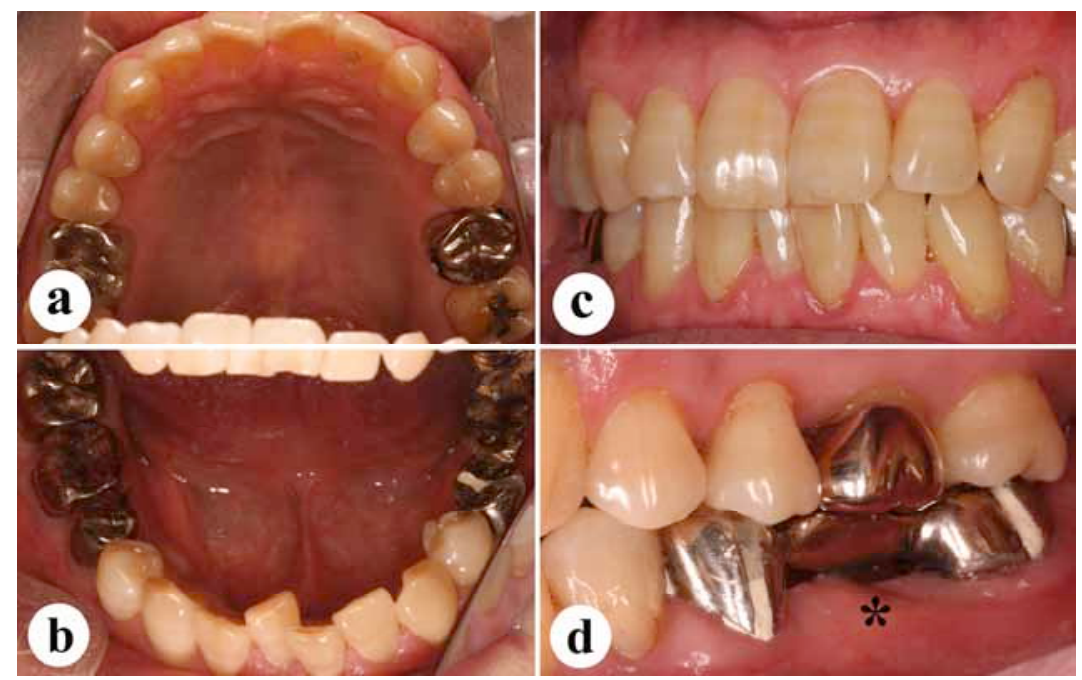

Fig. (1). Preoperative intraoral findings.

a: Upper dentition (occlusal view).

b: Lower dentition (occlusal view).

c: Front teeth (front view).

d: Left-side view of molar occlusal status. Planned implant site indicated with an asterisk (*).

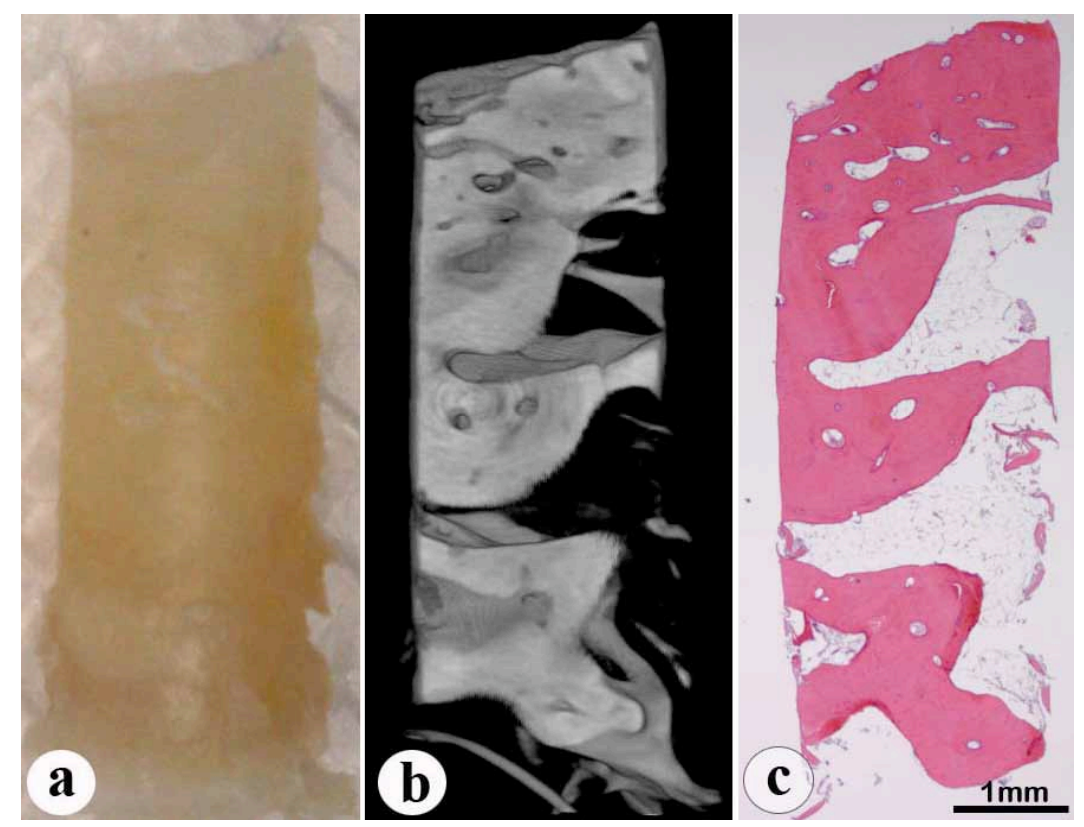

Fig. (2). Alveolar bone findings.

a: Appearance of the alveolar bone sample.

b: 3D-microCT image of the longitudinal section, through the center of the sample.

c: Hematoxylin-eosin staining of the same section as in (b).

Before surgery, periapical radiographs, panoramic radiograph, and CT images of the mandible were taken. The CT scanning was performed at the following settings: slice thickness $1.0 \mathrm{~mm} ; 120 \mathrm{kV}$ and $100 \mathrm{~mA}$ (Xvigor Real; Toshiba Medical Systems Co., Ltd., Tochigi, Japan). Bone turnover markers, including serum bone-specific alkaline phosphatase (BAP), serum osteocalcin (OC), serum type I collagen cross-linked N-telopeptide (NTX) and urinary deoxypyridinoline (DPD) were examined as part of the biochemical testing of the patient's systemic bone metabolism. A calcaneal quantitative ultrasound measurement was performed using Speed of Sound (SOS) with an ultrasound device (CM-200; Furuno Electric Co., Ltd., Hyogo, Japan).
Using a trephine bur, an alveolar bone sample (columnshaped; $2 \mathrm{~mm}$ in diameter, $7 \mathrm{~mm}$ in height) was obtained from the implant site (Fig. 2a), before the the implant was placed $(5 \mathrm{~mm}$ in diameter, $13 \mathrm{~mm}$ in height, Brånemark system ${ }^{\circledR}$, Nobel Biocare, Gothenburg, Sweden). The sample was fixed in $4 \%$ formaldehyde solution for 24 hours.

After fixation, the fine trabecular structures of the sample were observed with microCT (Fig. 2b), at the following settings: slice thickness $20 \mu \mathrm{m}$; magnification $4.58 \mathrm{X}$; $80.0 \mathrm{kV}$ and $76 \mu \mathrm{A}$ (ELE SCAN; Nittetsu. Elex Co., Ltd., Tokyo, Japan). The sample was then decalcified, embedded in paraffin and sliced into thin sections for hematoxylin-eosin stain- 


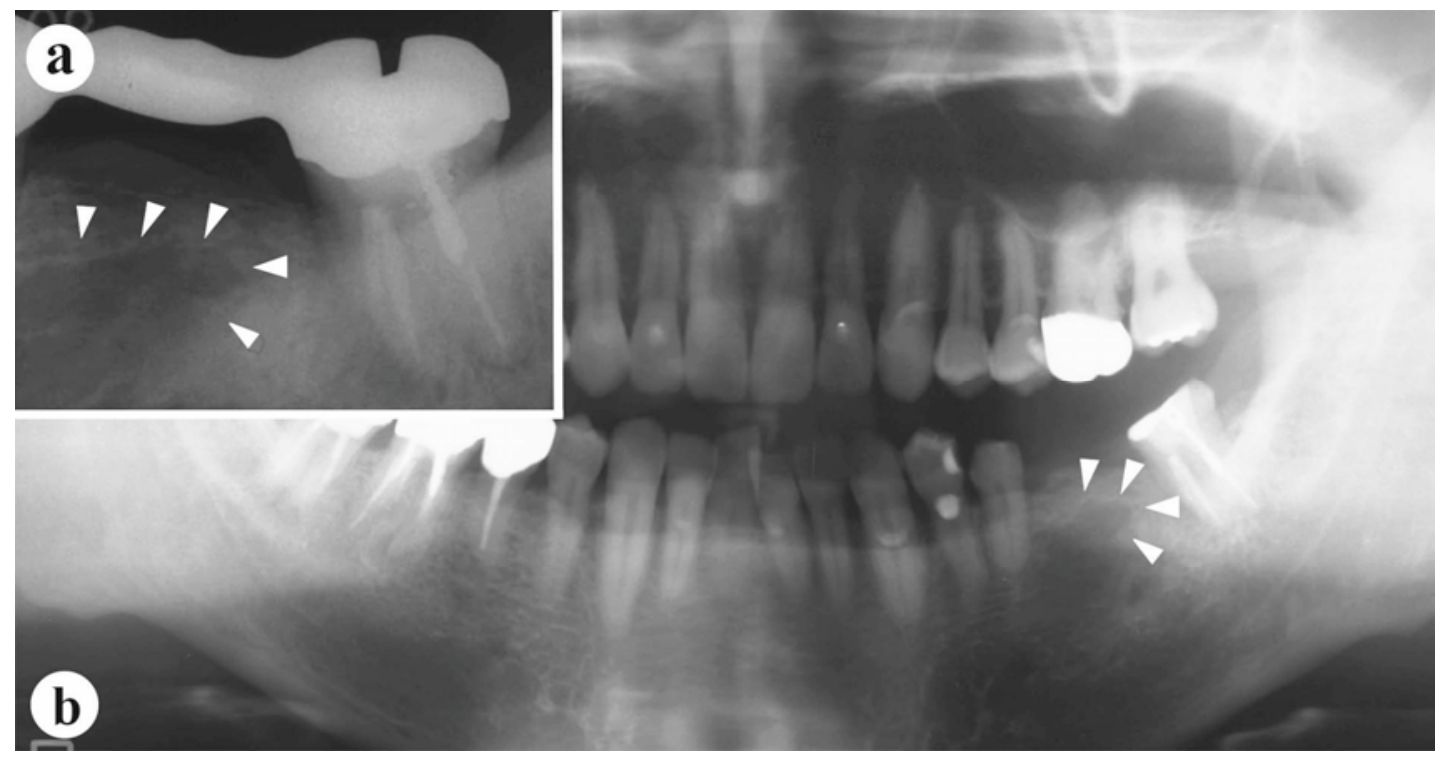

Fig. (3). Preoperative radiographic findings.

a: Periapical radiographof the planned implant site. Arrowheads indicate the borders of an irregularly-shaped radiolucency.

b: Panoramic radiograph, with an irregularly-shaped radiolucency (arrowheads) also visible.

Table 1. Bone Turnover Markers and Calcaneal Speed of Sound (SOS)

\begin{tabular}{|c|c|c|c|}
\hline \multicolumn{1}{|c|}{ Parameters } & Patient's Data & Normal Range \\
\hline \hline \multirow{3}{*}{$\begin{array}{c}\text { Bone turn- } \\
\text { over markers }\end{array}$} & BAP (IU/l) & 24.9 & $13.0-33.9$ \\
\cline { 2 - 4 } & OC (ng/ml) & 3.4 & $3.1-12.7$ \\
\cline { 2 - 4 } & NTX (nmolBCE/L) & 10.6 & $9.5-17.7$ \\
\hline & DPD (nmol/mmol $\cdot \mathrm{CRE})$ & 2.5 & $2.1-5.4$ \\
\hline
\end{tabular}

BAP: bone-specific alkaline phosphatase, OC: osteocalcin, NTX: type I collagen cross-linked N-telopeptide, DPD: deoxypyridinoline.

All bone turnover markers and calcaneal SOS were in normal ranges.

ing (Fig. 2c) and tartrate-resistant acid phosphatase (TRAP) staining. Bone histomorphometry was performed on three selected longitudinal sections, one through the center of the sample and the other two $0.5 \mathrm{~mm}$ from the central one, using bone histomorphometry system software (System Supply Co. Ltd, Nagano, Japan), and measurements were made of the following parameters: bone volume (\%), trabecular thickness $(\mu \mathrm{m})$, osteoblast surface $(\%)$, eroded surface $(\%)$, quiescent surface $(\%)$, trabecular number $(\mathrm{N})$, trabecular separation $(\mu \mathrm{m})$, erosion depth $(\mu \mathrm{m})[11]$. All data for the three sections was expressed as means and standard deviations.

The patient enrolled in the study received a verbal and written explanation of its goals and procedures, and subsequently signed an informed consent form, prior to participating in the study. All the procedures involved had already been approved by the Niigata University Ethics Committee (20-R13-08-06).

The panoramic and periapical radiographs revealed an irregularly-shaped radiolucency under the alveolar crest at the planned implant site (Fig. 3), and the trabeculae were not clearly visible with ordinary CT (Fig. 4). Blood and urine analyses indicated that the patient's bone metabolism was within the normal range (Table 1). The calcaneal bone density was slightly lower than the average for the same age group, but still within the standard deviation (SD) range (Table 1). MicroCT three-dimensional (3D) images of the bone sample clearly showed several plate-like trabeculae extending outward from the lingual cortical bone (Fig. 5). These trabeculae were poorly connected and widely separated.

Histological observations revealed that the marrow space was filled with adipose tissue (Fig. 6a), and that most of the bone surface was covered with flattened osteoblasts (Figs. $\mathbf{6 a}, \mathbf{c})$. However, some hematopoietic cells were observed in the bone marrow (Fig. 6b), and cuboidal osteoblasts were also seen on the bone surface in a few regions (Fig. 6d). A small number of TRAP-positive osteoclasts and cement lines were also observed (Figs. 6e, f). The histomophometricdata are shown in Table 2.

The dental implant is still functioning well, with no bone loss detectable on X-rays (Fig. 7) and no clinical problems two years post-operatively (Fig. 8).

\section{DISCUSSION}

Several thin trabeculae extending outward from the lingual cortical bone were clearly visible on the microCT images of the sample taken from the implant site, although the trabecular structure was unclear in the CT images obtained 


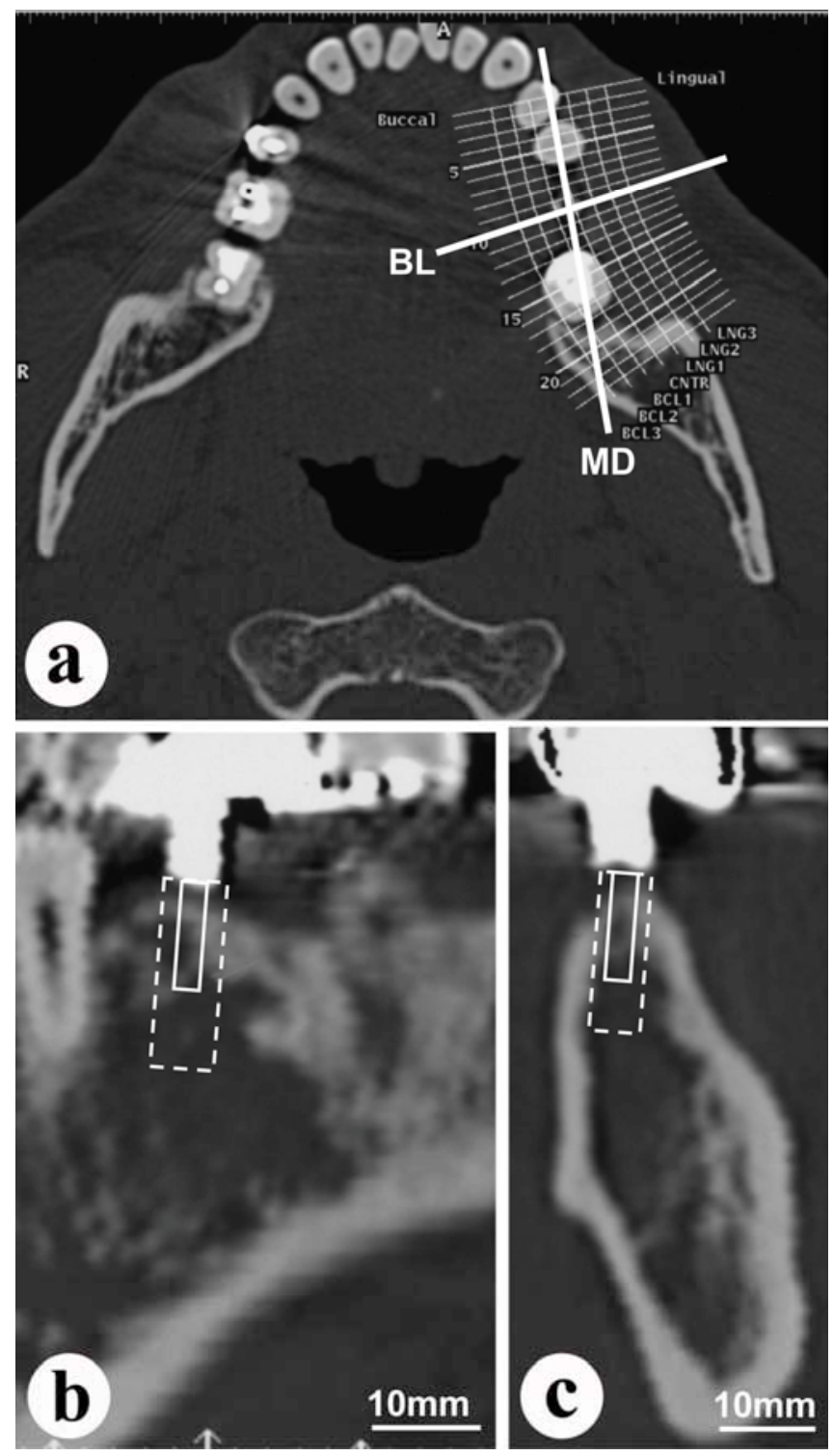

Fig. (4). Conventional CT images.

a: Horizontal section at the height of the patient's dental crowns. (MD: mesio-distal line; BL: bucco-lingual line, through the planned implant site).

b: Mesio-distal section at the MD line in (a) above. Dotted line indicates planned implantation site. Solid line indicates planned bone biopsy site. c: Bucco-lingual section at the BL line in (a) above. Dotted line indicates planned implantation site. Solid line indicates planned bone biopsy site.

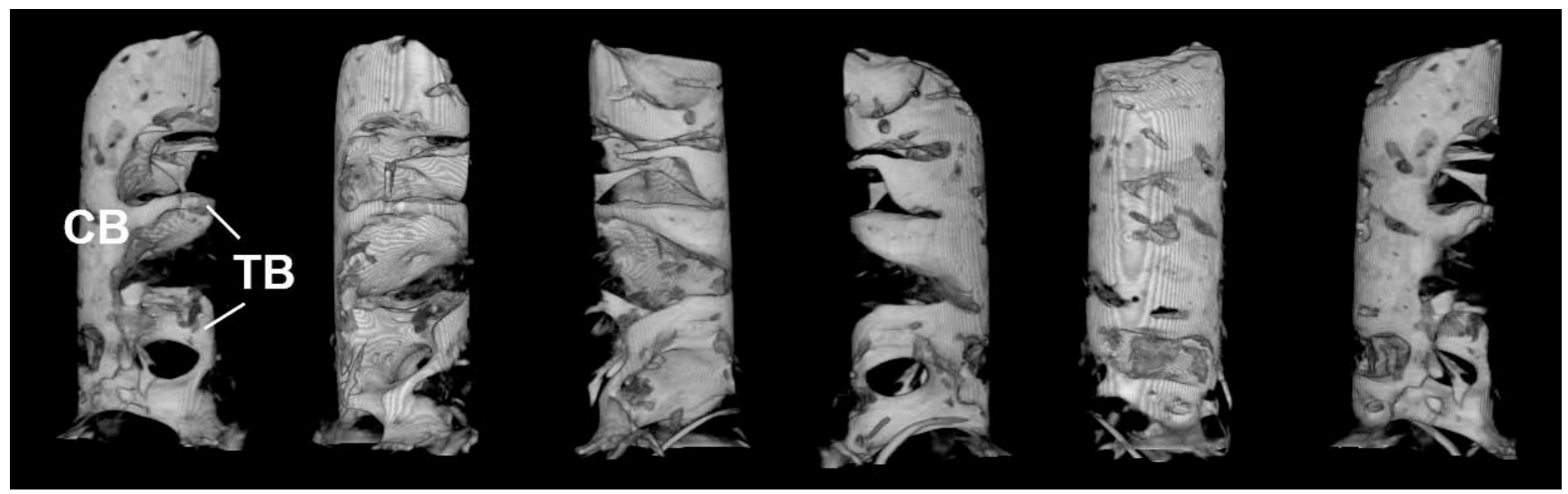

Fig. (5). 3D-microCT images of the alveolar biopsy sample.

Left to right: microCT images, with the sample being successively rotated approx. 60 degrees clockwise each time.

CB: cortical bone; TB: plate-like trabeculae. 


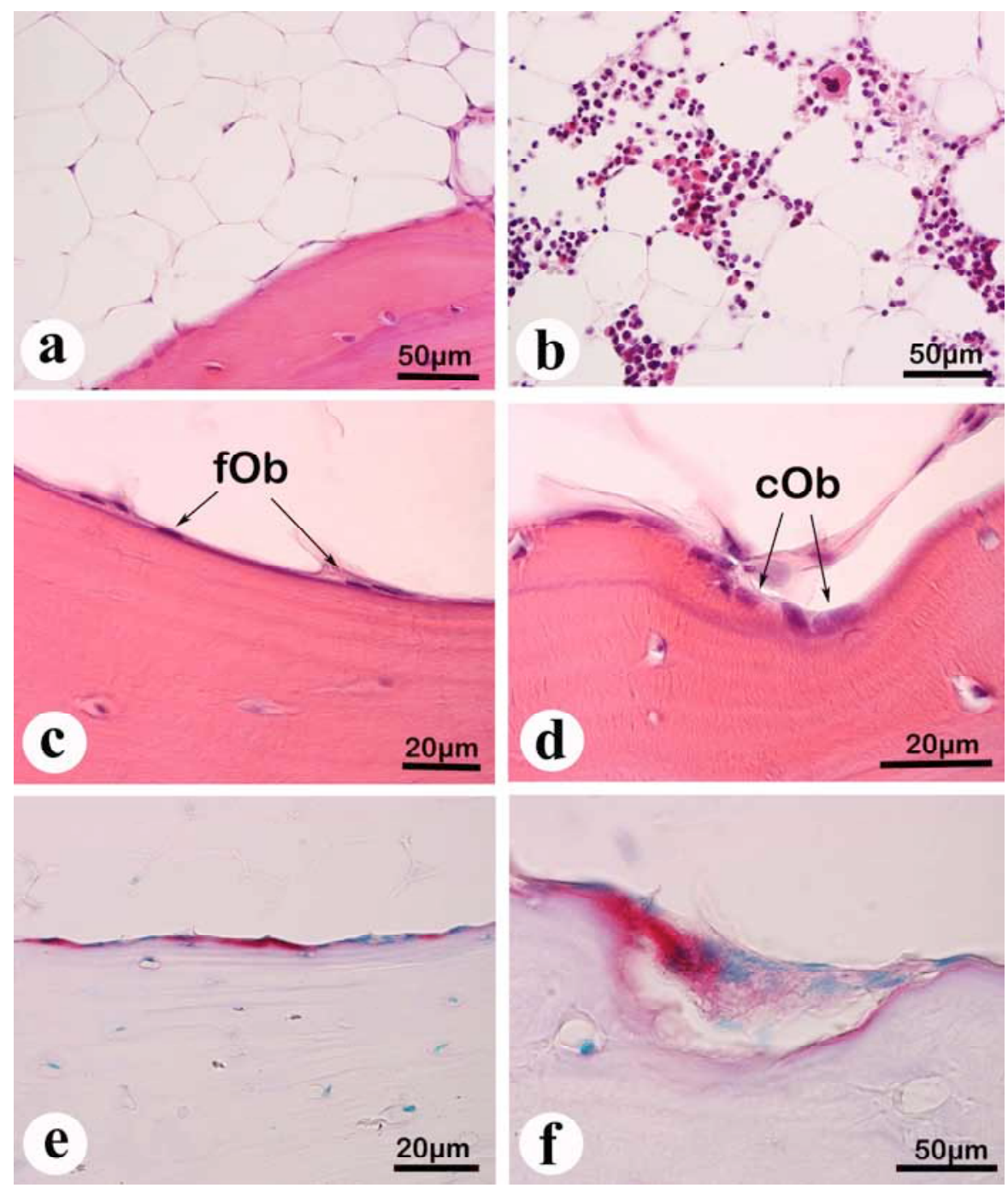

Fig. (6). Histological findings.(a) to (d) Hematoxylin-eosin staining of paraffin sections. (e) and (f) TRAP staining of paraffin sections.

a: Adipose tissue, visible over a wide range in the marrow space.

b: Hematopoietic cells, occasionally visible in certain areas of the marrow.

c: Several flattened osteoblasts (fOb), visible at the bone surface.

d: Cuboidal osteoblasts (cOb), occasionally visible at the bone surface.

e: TRAP-positive bone surface, indicated by the red staining at the bone surface.

f: A few TRAP-positive osteoclasts, on the resorption lacuna.

Table 2. Results of Bone Histomorphometry

\begin{tabular}{|c|c|c|c|c|}
\hline Parameter & Unit & Mean & \pm & SD \\
\hline Trabecular thickness & (um) & 335.15 & \pm & 55.46 \\
\hline Osteoblast surface & $(\%)$ & 14.85 & \pm & 2.97 \\
\hline Eroded surface & $(\%)$ & 1.95 & \pm & 0.44 \\
\hline Trabecular number & $(\mathrm{N})$ & 1.57 & \pm & 0.28 \\
\hline Trabecular separation & (um) & 314.66 & \pm & 169.70 \\
\hline Erosion depth & (um) & 4.71 & \pm & 0.91 \\
\hline
\end{tabular}



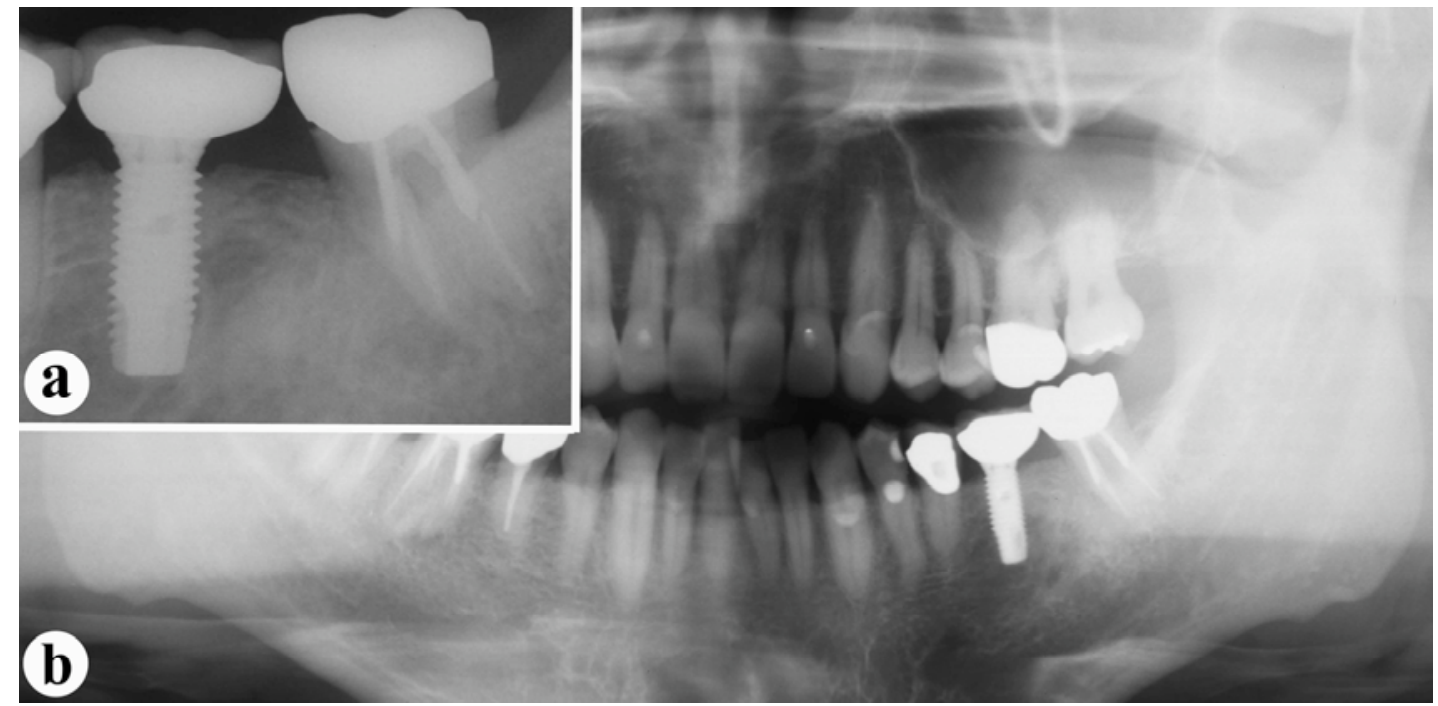

Fig. (7). Radiographic findings, two years post-operatively.

a: Periapical radiograph of planned implant site.

b: Panoramic radiograph.

Although a radiolucent area is still visible around the center of the implant, bone is also visible directly adjacent to the implant.
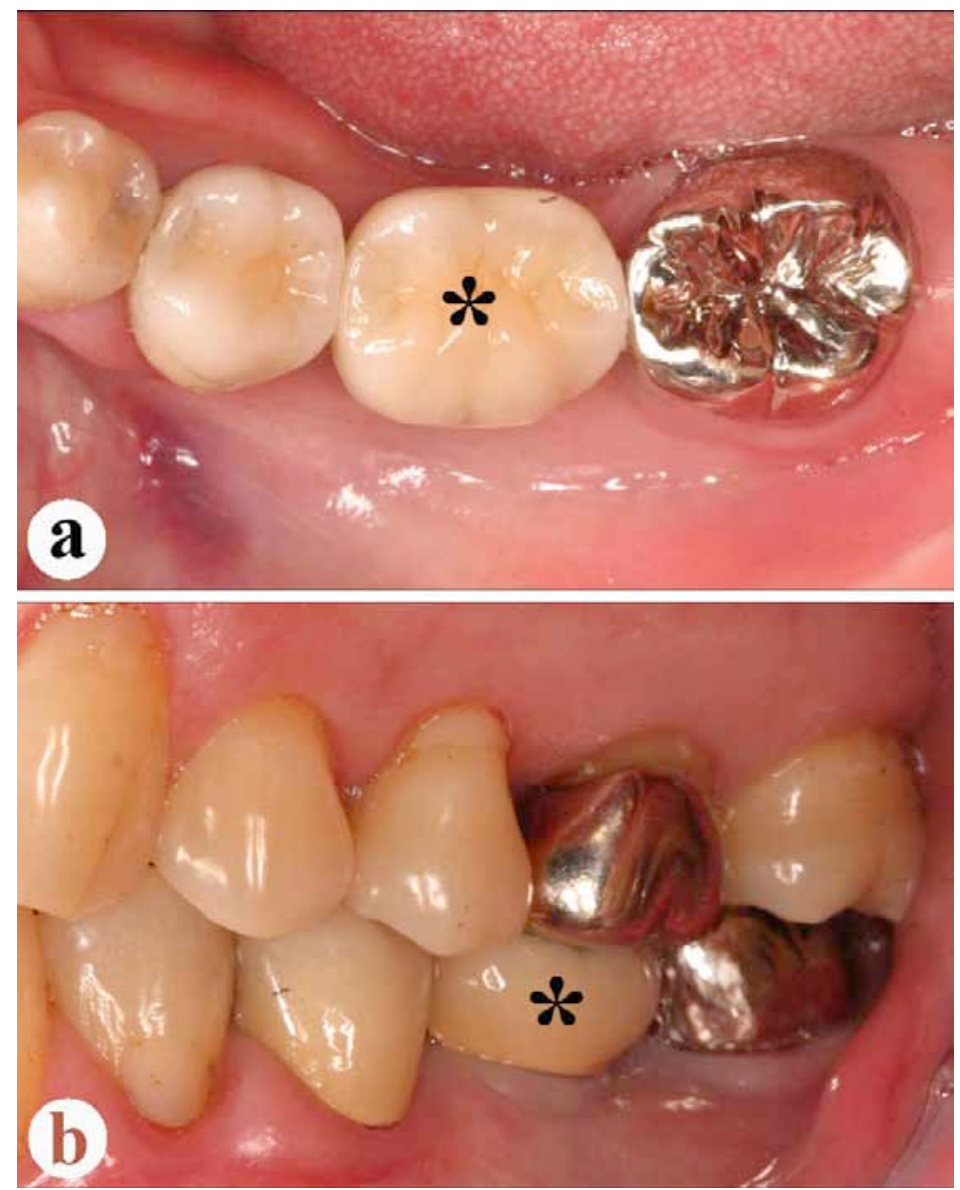

Fig. (8). Intraoral findings, two years post-operatively.

a: Occlusal view. Ceramic crown (asterisk) set onto the implant.

b: Lateral view at occlusion. Asterisk indicates same ceramic crown. No abnormal findings are clinically visible in the implant area.

with conventional CT. This led us to expect that the implant would have enough direct contact with the cortical and trabecular bone surfaces for sufficient primary stability. The

existence of cuboidal osteoblasts, together with a small number of osteoclasts, also underlined the occurrence of bone remodeling in this region. 
The presence of marrow spaces containing mesenchymal cells, together with blood cells surrounding the implant surfaces, have been reported to be of great importance in achieving implant osseointegration [12]. In our patient's alveolar bone, although the marrow space was mostly occupied with adipose tissue, blood cells were also found in small areas of the marrow. We were therefore able to expect sufficient osseointegration would be achieved between bone and implant [8], and in fact, the implant has actually been functioning well for two years since its insertion.

Calcaneal SOS and bone turnover markers of our patient were all within normal ranges. This would indicate that this patient's systemic bones were in normal condition, and that he was not at risk for osteoporotic fractures: calcaneal SOS in particular has been reported to be an effective predictor of the risk of osteoporotic fracture [13]. Histological observation indicated that his alveolar bone status was also normal. Although the present study is a case report, it would seem to suggest the more general possibility of predicting a patient's systemic bone condition using alveolar bone biopsy. If histomorphometric data for many alveolar bone samples could be gathered and compared with the systemic bone metabolic markers and/or bone mineral densities of the same subjects, clinically-useful diagnostic reference values for alveolar bone histomorphometry might be established. Recently, some studies have tried to determine the presence of osteoporosis based on minute alveolar bone changes [14-16]. In the future, more data will be necessary in order to establish clinical guidelines for when these microCT procedures would be recommended, and when they would be unnecessary, due to the presence on conventional medical CT and/or CBCT images of sufficient alveolar bone trabeculae. More data would also allow for the creation of morphometric standards for quantifying the bone quality required for osseointegration to occur, and resist the loading stresses arising from mastication.

Due to the invasive nature of bone biopsies done on ribs or iliac bones, it is not easy to convince healthy subjects to undergo them. However, it is relatively simple to use an alveolar bone tissue extracted as part of the implant cavity preparation process, for the purpose of biopsy samples, with few ethical problems involved as well. It must be admitted that the alveolar bone sample extracted during the implant operation is smaller than samples obtained from the iliac bone or rib. On the other hand, such invasive biopsies involving the rib or iliac bone may be avoidable thanks to this procedure, as sufficient information about systemic bone metabolism may be obtainable from the alveolar bone sample.

Another limitation of the procedure discussed in this case study is that the alveolar bone sample can only be analyzed once the operation has been carried out. For this reason, such samples cannot be used in making a decision about the initial viability of the implant treatment: for this purpose, conventional techniques involving CT imaging of the alveolar bone are generally used. However, once the operation has been done and the sample obtained, the information obtained from it could be useful for the clinical evaluation of patients' systemic bone conditions, as well as predicting the success of implant osseointegration.

\section{CONCLUSION}

An alveolar bone biopsy sample was obtained from a 53year-old male patient's mandibular bone during implant cavity preparation. Important information was directly obtained from this sample using microCT and histological and histomorphometric techniques, such as the existence of thin trabeculae and several active bone cells. Such a detailed understanding of the condition of the bone at a planned implant site, obtained prior to the insertion of the implant, would therefore seem to be useful in predicting its successful osseointegration.

\section{CONFLICT OF INTERESTS}

The authors confirm that this article content has no conflicts of interest.

\section{ACKNOWLEDGEMENT}

This work was supported by a Grant-in-Aid for Research Activity Start-up from JSPS (No. 23890061). The authors also wish to thank Associate Professor Ian Megill of Niigata Seiryo University, for his assistance in preparing the manuscript.

\section{REFERENCES}

[1] Jaffin RA, Berman CL. The excessive loss of Branemark fixtures in type IV bone: a 5-year analysis. J Periodontol 1991; 62: 2-4.

[2] Herrmann I, Lekholm U, Holm S, Kultje C. Evaluation of patient and implant characteristics as potential prognostic factors for oral implant failures. Int J Oral Maxillofac Implants 2005; 20: 220-30.

[3] Malik R, Garg R, Suresh DK, Chandna S. Success or failure of a dental implant: its relationship to bone density: a case report of a failed implant. J Contemp Dent Pract 2010; 11: E065-72.

[4] Lekholm U, Zarb G. Patient selection and preparation. Tissueintegrated prostheses: Osseointegration in clinical dentistry. Chicago: Quintessence Publishing 1985; pp. 199-209.

[5] Yim JH, Ryu DM, Lee BS, Kwon YD. Analysis of digitalized panorama and cone beam computed tomographic image distortion for the diagnosis of dental implant surgery. J Craniofac Surg 2011; 22: 669-73.

[6] Lindh C, Petersson A, Rohlin M. Assessment of the trabecular pattern before endosseous implant treatment: diagnostic outcome of periapical radiography in the mandible. Oral Surg Oral Med Oral Pathol Oral Radiol Endod 1996; 82: 335-43.

[7] Ribeiro-Rotta RF, Lindh C, Rohlin M. Efficacy of clinical methods to assess jawbone tissue prior to and during endosseous dental implant placement: a systematic literature review. Int J Oral Maxillofac Implants 2007; 22: 289-300.

[8] Nkenke E, Hahn M, Weinzierl K, Radespiel-Troger M, Neukam FW, Engelke K. Implant stability and histomorphometry: a correlation study in human cadavers using stepped cylinder implants. Clin Oral Implants Res 2003; 14: 601-9.

[9] Trisi P, Rao W. Bone classification: clinical-histomorphometric comparison. Clin Oral Implants Res 1999; 10: 1-7.

[10] Ribeiro-Rotta RF, Lindh C, Pereira AC, Rohlin M. Ambiguity in bone tissue characteristics as presented in studies on dental implant planning and placement: a systematic review. Clin Oral Implants Res 2011; 22: 789-801.

[11] Parfitt AM, Drezner MK, Glorieux FH, et al. Bone histomorphometry: standardization of nomenclature, symbols, and units. Report of the ASBMR Histomorphometry Nomenclature Committee. J Bone Miner Res 1987; 2: 595-610.

[12] Marco F, Milena F, Gianluca G, Vittoria O. Peri-implant osteogenesis in health and osteoporosis. Micron 2005; 36: 630-44

[13] Turner CH, Peacock M, Timmerman L, Neal JM, Johnson CC, Jr. Calcaneal ultrasonic measurements discriminate hip fracture independently of bone mass. Osteoporos Int 1995; 5: 130-5. 
[14] Arisaka R, Matsuo A, Chiba H, et al. Preliminary clinical study to evaluate the relationship between systemic bone turnover and the microstructure of the alveolar bone. Oral Sci Int 2009; 6: 27-35.

[15] Ratiu CA, Miclaus V, Maghiar T, Ratiu IA, Osvat D, Ober C. The utility of histopathologic examination in appreciation of mandibular osteoporotic status. Rom J Morphol Embryol 2010; 51: 467-72.
[16] Yamashita-Mikami E, Tanaka M, Sakurai N, et al. Correlations between alveolar bone microstructure and bone turnover markers in pre- and post-menopausal women. Oral Surg Oral Med Oral Pathol Oral Radiol Endod 2011 (in press).

Received: February 27, 2013

Revised: March 08, 2013

Accepted: March 11,2013

(c) Yamashita-Mikami et al.; Licensee Bentham Open.

This is an open access article licensed under the terms of the Creative Commons Attribution Non-Commercial License (http://creativecommons.org/licenses/by-nc/3.0/) which permits unrestricted, non-commercial use, distribution and reproduction in any medium, provided the work is properly cited. 